\title{
ANALYTICAL MODELING OF INTERFERENCE IN CELLULAR FIXED RELAY NETWORKS
}

\author{
Sebastian S. Szyszkowicz, Halim Yanikomeroglu, Eman Fituri, Shalini Periyalwar \\ Department of Systems and Computer Engineering, Carleton University \\ email: \{sz, halim, efituri\}@sce.carleton.ca, speriyalwar@hotmail.com
}

\begin{abstract}
We develop a simple yet accurate analysis of the interference distribution in a cellular system, with particular emphasis on a two-hop fixed relay network, though the analysis may apply to much wider contexts. Similar analyses have already been proposed, but suffer from being too specific in their assumptions, are analytically difficult, consider only the uplink, and are not necessarily validated. We provide a simple closed-form solution for a wide variety of cases and validate all our theoretical curves directly by Monte-Carlo simulations of the exact same models.

Our method is flexible for many channel and system parameters, and for arbitrary cellular layouts, thus it can readily be applied to a two-hop relay context.
\end{abstract}

Keywords: Interference in cellular networks, fixed relay networks, downlink interference, sum of lognormal variants

\section{Introduction}

When modelling interference in cellular communications we encounter the problem of finding the distribution of the sum of several (not necessarily independent or identically distributed) lognormal random variables (RV's) [1-4]. Indeed, the power received from an interferer in a wireless context, when averaged over time and position, can be well modeled by a lognormal RV, even when other factors multiply the power received from each interferer (Rayleigh fading, pathloss) [4]. In particular, the downlink analysis in cellular systems has been considered problematic, since it involves the sum of correlated random variables [6]. We propose an approximate solution which consists in finding the exact moments of the interference power. The moments are found using an original method, where we decouple the layout of the interfering cells from the other system parameters. Our method is general, and might solve other problems that include sums of correlated RV's. We match the obtained moments to those of a lognormal distribution, which is often used to model the total interference in cellular systems $[2,3]$. We multiply the result by a Bernoulli $\mathrm{RV}$, to correct for low-loading conditions.

This is not the most exact method, because the exact distribution of the sum of lognormal RV's is not perfectly lognormal [1], and because matching the first two moments will not necessarily yield the best-fitting lognormal distribution [2]. Our method, however, has the advantage of being analytically simple and flexible. Indeed, we give an analysis for the downlink that we believe to be new, usually simulation being required $[5,6]$, while also solving for the uplink. Unlike [4], we cover cases with and without power control. Also, unlike the Schwartz \& Yeh method, which is iterative [3,4], we can obtain direct analytical expressions. Furthermore, we incorporate user activity as a Bernoulli RV, which is not part of the analysis in [3], and which is also pertinent to frequencyhopping cases such as [4-6]. Our method applies to irregular geometries, such as used in simulations in [5].

We describe our system model in section 2, provide an analysis in section 3, validate our analytical results by MonteCarlo simulations in section 4 for both conventional and relay cellular networks, and present our conclusions in section 5 .

\section{Universal System Model}

We would like to propose a system model that is as general as possible, while retaining enough simplicity for analytical tractability. The model covers the cases in [4-6], and more.

\subsection{Conventional Cellular Layout}

We assume that all the cells are identical regular hexagons of unit side length, each with its base station (BS) in the center, as shown in figure 1. A mobile user (MU) is assumed to be connected to its nearest BS, i.e. it lies in the corresponding hexagon. The position of the MU is assumed random, uniformly distributed over the cell. We call the cell under consideration $C_{0}$, and all potentially interfering cells $C_{i}$.

\subsection{Fixed Relaying}

We consider a two-hop fixed relaying scenario [5]. We define an augmented cell as the combination of a central subcell containing a base station and its surrounding six sub-cells containing relays. The six relays each have a good orthogonal wireless link with their base station. There is no communication between augmented cells, and we can assume that the interference from all sub-cells within the augmented cell has been adequately managed [5] and is negligible, while all other sub-cells are considered as interferers. We find the interference arriving at a sub-cell serviced by a relay, which is described by the geometry in figure 3 .

\subsection{Channel Assignment}

We assume that each MU within a sub-cell uses an orthogonal channel. Consequently, there is only intercellular 
interference, and there can be at most one interferer in each sub-cell $C_{i}$. Thus, the presence of interference from a particular sub-cell $C_{i}$ can be modeled by a Bernoulli RV with parameter $p$ (collision probability), which is a function of loading, number of channels, channel reuse, DTX [6], etc.

\subsection{Uplink and Downlink}

In the uplink $\left(k_{D L}=0\right)$, the $\mathrm{BS}$ of $C_{0}$ can receive interference signals from MU's in other sub-cells. In the downlink $\left(k_{D L}=1\right)$, it is the MU under analysis, located in $C_{0}$, that receives interference from other BS's, which are transmitting to their own MU's. The marginal distribution of the interference power from a given sub-cell $i$ is identical for uplink and downlink. However, only for the uplink are the pathlosses independent for each interferer.

\subsection{Signal Fading}

The signal coming from an interferer experiences Rayleigh fading (though other fading models could just as well be employed). The interfering signal also suffers long-term shadowing, which follows a lognormal law, with $\sigma(\mathrm{dB})=6$ to $12 \mathrm{~dB}$ spread. All paths are assumed to be independently faded and shadowed. Throughout the paper, we use the natural base for lognormal RV's. Thus we convert from decibel units (typically used for shadowing) to natural units by multiplying by a factor of $\lambda=\ln (10) / 10 \approx 0.23$. In natural units, $\sigma=\lambda \sigma(\mathrm{dB})$.

\subsection{Optional Power Control}

Without loss of generality, we assume that, without power control $\left(k_{P C}=0\right)$, all interferers are transmitting at unit power. Under power control $\left(k_{P C}=1\right)$, the power $P_{i}$ transmitted by the $i^{\text {th }}$ interferer, has identical statistics for uplink and downlink and will be affected by: the pathloss between the interfering MU-BS pair, the lognormal shadowing with spread $\sigma$ and the power control lognormal error with spread $\sigma_{e}(\mathrm{~dB})=0$ to $1 \mathrm{~dB}$.

\section{Analysis}

This analysis shows how the first two moments of a sum of correlated RV's can be found exactly, given that the said RV's can be separated into independent, identically distributed (i.i.d.) and correlated components. Once the moments are found, we proceed to match them to a lognormal RV multiplied by a Bernoulli RV, which has known moments.

\subsection{Problem Statement}

The total interference received, for any of the possible system configurations described in section 2, can be written as:

$$
I=\sum_{i=1}^{N} I_{i}=\sum_{i=1}^{N} R_{i} \zeta_{i} \chi_{i} \alpha_{i}^{2} P_{i}
$$

Here there are $N$ potential interferers (equivalently, $N$ cells or sub-cells), and $I_{i}$ is the interference produced by sub-cell $i$. $R_{i}$ is the pathloss to the interferer and is equal to $r_{i}^{-\beta}$, where $r_{i}$ is the physical normalised distance between the interferer $i$ and the desired point. In general, $R_{i}$ 's have different distributions, and are not independent when considering the downlink. $\zeta_{i}$ and $\alpha_{i}^{2}$ represent shadowing and fading respectively between the interferer $i$ and the receiver. $\chi_{i}$ is the Bernoulli-distributed indicator function that is unity when there is an active user in interfering sub-cell $i$ on the channel under consideration, otherwise zero. $P_{i}$ is the (normalised) transmit power of the interferer in sub-cell $i$.

\subsection{Finding the First Two Moments Exactly}

In order to find moments of the sum in (1), we group the random variables as follows:

$$
Q_{i}=\zeta_{i} \chi_{i} \alpha_{i}^{2} P_{i} \Rightarrow I=\sum_{i=1}^{N} R_{i} Q_{i}
$$

Thus we separate the interference into two components: $R_{i}$ 's, which are, in general, of different distributions and not independent, and the $Q_{i}$ 's, which are i.i.d RV's.

We define the $A$-coefficients as follows:

$$
\begin{aligned}
& A_{n}=E\left[\sum_{i=1}^{N} R_{i}^{n}\right] \\
& A_{D L}=E\left[\left(\sum_{i=1}^{N} R_{i}\right)^{2}\right] \\
& A_{U L}=\sum_{i=1}^{N}\left(E\left[R_{i}\right]\right)^{2}
\end{aligned}
$$

The expectation is taken over the position of the mobiles being uniformly distributed over their respective sub-cells.

Since $Q_{i}$ 's are i.i.d.:

$$
B_{n}=E\left[Q_{i}^{n}\right], \forall i
$$

Now we can express the exact moments of the interference:

$$
\begin{gathered}
E[I]=A_{1} B_{1} \\
E\left[I^{2}\right]= \begin{cases}A_{2} B_{2}+\left(A_{1}^{2}-A_{U L}\right) B_{1}^{2} & k_{D L}=0 \\
A_{2} B_{2}+\left(A_{D L}-A_{2}\right) B_{1}^{2} & k_{D L}=1\end{cases}
\end{gathered}
$$

\subsection{Obtaining the $A$-coefficients}

We can rewrite the $A_{n}$ and $A_{U L}$ coefficients as:

$$
\begin{gathered}
A_{n}=\sum_{i=1}^{N} E\left[r_{i}^{-n \beta}\right]=\sum_{i=1}^{N} \kappa_{i}(n \beta) \\
A_{U L}=\sum_{i=1}^{N} \kappa_{i}^{2}(n \beta) \\
\kappa_{i}(n \beta)=\frac{2}{3 \sqrt{3}} \iint_{(x, y) \in C_{0}}{\sqrt{\left(x-x_{i}\right)^{2}+\left(y-y_{i}\right)^{2}}}^{-n \beta} d x d y
\end{gathered}
$$

Where $C_{0}$ is a hexagon of unit side (its area is $3 \sqrt{3} / 2$ ) centered at $(0,0)$ with the proper orientation, and $\left(x_{i}, y_{i}\right)$ are the coordinates of the interfering base station $i$. These integrals can be evaluated separately for each interferer, and then summed for all interferers. Similar integrals can be found in [6]. 
In the downlink, the distances between a mobile and the interfering fixed base stations are not independent, and $A_{(D L)}$ cannot be separated into a sum of terms, but must be integrated for the entire geometry as follows:

$$
A_{D L}=\frac{2}{3 \sqrt{3}} \iint_{(x, y) \in C_{0}}\left(\sum_{i=1}^{N} \sqrt{\left(x-x_{i}\right)^{2}+\left(y-y_{i}\right)^{2}}\right)^{-\beta} d x d y
$$

Where $x_{i}, y_{i}$ and $C_{0}$ are the same as in (11).

In table 1 , we calculate $A$-coefficients for all our scenarios.

\subsection{Obtaining the $B$-coefficients}

Since the components of $Q_{i}$ are independent, and all $Q_{i}$ are i.i.d., the $B$-coefficients have general form:

$$
B_{n}=E\left[Q_{i}^{n}\right]=E\left[\zeta_{i}^{n}\right] E\left[\alpha_{i}^{2 n}\right] E\left[\chi_{i}^{n}\right] E\left[P_{i}^{n}\right], \forall i
$$

If there is no power control, then: $P_{i}=1, \forall i$.

With power control, the power transmitted will be proportional to the pathloss between the mobile $i$ and its base station. The position of the mobile has uniform distribution over its sub-cell area $C_{i}$. Now the sub-cell area is a regular hexagon of unit side, which it is conventional to approximate by a circle of radius $\rho \leq 1$. We define the mean pathloss between a mobile $i$ and its own base station to be $L_{i}$ :

$$
\begin{gathered}
L_{i} \sim f_{L}(x)=\frac{2}{\beta \rho^{2}} x^{\frac{2}{\beta}-1}, x \in\left[0, \rho^{\beta}\right] \\
E\left[L_{i}^{n}\right]=\frac{2}{2+n \beta} \rho^{n \beta}
\end{gathered}
$$

In $[4,6], \rho$ is assumed 1 . We found, through numerical integration, that $\rho=0.915$ gives a good approximation for the first two moments and use this value in our analysis.

The transmit power will also be affected by lognormal shadowing, for which the power control mechanism will attempt to compensate. We model this by a lognormal variable with statistics $\left(0, \sigma^{2}+\sigma_{e}^{2}\right)$, as in [4]. Finally, with power control, the moments of the transmit power are:

$$
E\left[P_{i}^{n}\right]=\frac{2}{2+n \beta} \rho^{n \beta} e^{\frac{n^{2}}{2}\left(\sigma^{2}+\sigma_{e}^{2}\right)}
$$

Consequently:

$$
B_{n}=p \cdot n ! e^{\frac{n^{2}}{2} \sigma^{2}}\left[\frac{2}{2+n \beta} \rho^{n \beta} e^{\frac{n^{2}}{2}\left(\sigma^{2}+\sigma_{e}^{2}\right)}\right]^{k_{P C}}
$$

\subsection{Matching the Moments}

We approximate $I$ by a lognormal RV of parameters $\left(a_{I}, b_{I}^{2}\right)$ multiplied by an independent Bernoulli RV with parameter $p_{\chi}$ :

$$
\begin{gathered}
E\left[I^{n}\right]=p_{\chi} \cdot e^{n a_{I}+\frac{n^{2}}{2} b_{I}^{2}} \\
p_{\chi}=P[I \neq 0]=1-(1-p)^{N}
\end{gathered}
$$

Inverting (18), we obtain:

$$
\begin{aligned}
& a_{I}=2 \ln E[I]-\frac{1}{2} \ln E\left[I^{2}\right]-\frac{3}{2} \ln \left(p_{\chi}\right) \\
& b_{I}^{2}=-2 \ln E[I]+\ln E\left[I^{2}\right]+\ln \left(p_{\chi}\right)
\end{aligned}
$$

$a_{I}, b_{I}^{2}$ and $p_{\chi}$ determine the final approximate distribution:

$$
F_{I}(x) \cong \Phi\left(\frac{\ln x-a_{I}}{b_{I}}\right) p_{\chi}+\left(1-p_{\chi}\right), x>0
$$

Here $\Phi(x)$ is the standard normal cumulative distribution function.

We have thus found an approximate distribution for the interference. We will proceed to show how it is a good approximation, by comparing with simulated curves.

\section{Simulation Results}

We purpose to validate our analytical results by MonteCarlo simulations of the exact same random model. In figures 1-3 we directly compare the analytical (dashed) curves and their corresponding simulated (solid) curves of the complementary cumulative distribution functions of the normalised interference: $P(I>x)=1-F_{I}(x)$ in log-log scale. We calculate the A-parameters for all the geometries used (table 1). We only examine the downlink interference, since it is considered more difficult analytically [6] and because for all the cases that we considered, the results for uplink and downlink (all other parameters being equal), were extremely close. This is a result already observed in [3]. Hence, all our curves can be considered valid for both uplink and downlink.

\subsection{Conventional Cellular Model}

We examine the behaviour of the downlink interference in a three-tier layout (figure 1). We consider the case when $\beta=4$, with imperfect power control, and vary the shadowing parameter over a typical range of 6 to $12 \mathrm{~dB}(\sigma=1.382$ to 2.763) (figure 1). We then consider a case with pathloss $\beta=3$ or 4, with and without imperfect power control, fixing shadowing at $8 \mathrm{~dB}(\sigma=1.842)$ (figure 2$)$. In both cases, we keep the power control error at $1 \mathrm{~dB}\left(\sigma_{e}=0.2303\right)$ and $p=0.1$.

\subsection{Relaying Model}

We consider a two-hop relay network with $\beta=4$, imperfect power control, and shadowing of $8 \mathrm{~dB}(\sigma=1.842)$. We vary $p$, which is proportional to loading. Theoretical and simulated results match very well in this case (figure 3 ). It is worth noting that for $p=1$, the loading would effectively be 7 [5] and transmission would be continuous, because each channel would always be used 7 times per augmented cell i.e. per BS. This would be an extreme case, and values of $p<0.5$ are more practical. Our approximation has the closest matches for the relaying geometry and smaller values of $p$.

Table 1. A-parameters for various layouts and $\beta$.

\begin{tabular}{|c|c|c|c|}
\hline Layout & \multicolumn{2}{|c|}{ Conventional (figure 1) } & Relay (figure 3) \\
\hline $\boldsymbol{\beta}$ & 3 & 4 & 4 \\
\hline $\boldsymbol{A}_{\mathbf{1}}$ & 2.2582 & 1.4867 & 0.7935 \\
\hline $\boldsymbol{A}_{\mathbf{2}}$ & 1.0274 & 0.9830 & 0.4921 \\
\hline $\boldsymbol{A}_{\boldsymbol{D L}}$ & 5.2800 & 2.5260 & 0.9932 \\
\hline $\boldsymbol{A}_{\boldsymbol{U} \boldsymbol{L}}$ & 0.4860 & 0.2880 & 0.1444 \\
\hline
\end{tabular}






Figure 1. Varying the shadowing parameter in a conventional cellular network with imperfect power control.

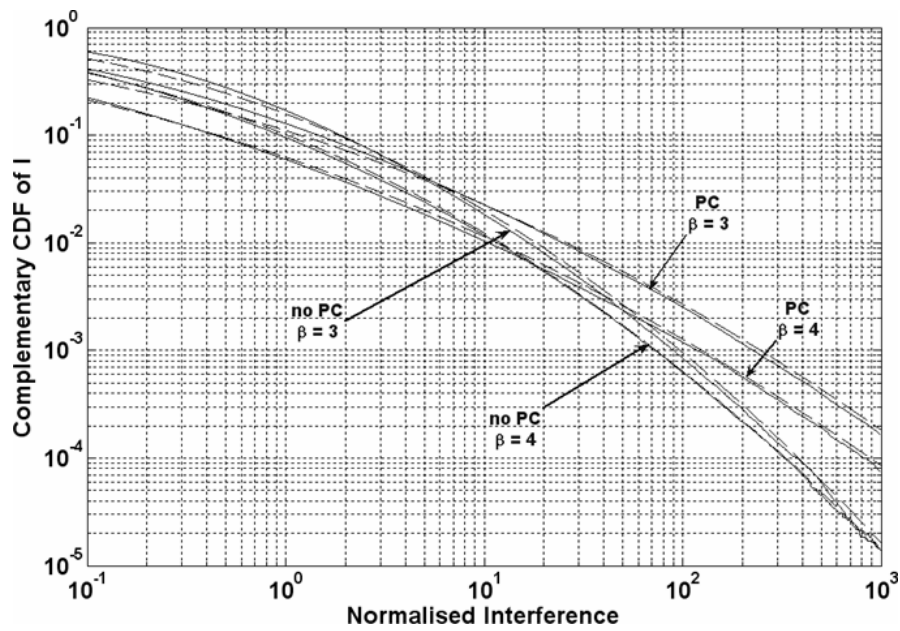

Figure 2. Varying the power control and propagation constant in a conventional cellular network.

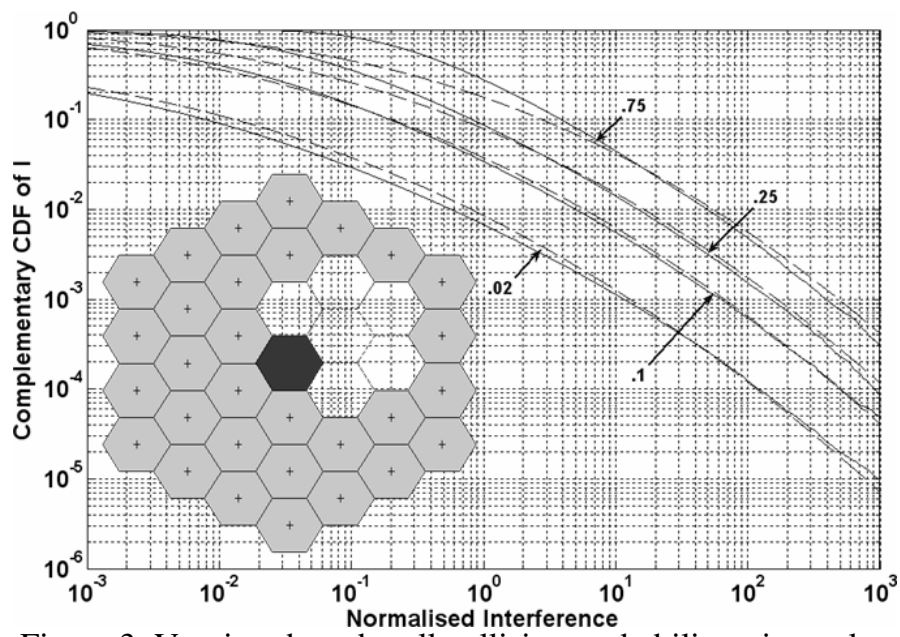

Figure 3. Varying the sub-cell collision probability $p$ in a relay network with imperfect power control.

\section{Conclusion}

In our analysis, we were able to find exact closed-form expressions for the first two moments of the interference in a cellular system while varying many useful parameters over realistic ranges. The numerical integrations required depend only on the positions of interfering cells and the propagation constant. Consequently, they can be calculated once and tabulated, while factors such as shadowing, fading and user activity are varied independently. Our method is very general and potentially encompasses other channel models: for example, Rayleigh fading could easily be replaced by another fading model.

Our simulation results show that this method is particularly well-suited for analysing the interference in a relay context, given the irregular geometry and lower probability of collision $p$ for each sub-cell. The method also gives a good approximation for the interference in a conventional cellular network.

Various frequency hopping schemes are currently being proposed for fixed relay networks, where channel reuse factors of more than one per base station could be envisioned [5] thanks to the interference averaging properties of these schemes $[4,5]$. We propose our method as a tool for analysing the interference in such systems, in order to better understand the interference dynamics, and, ultimately, to design better interference mitigation techniques.

\section{Acknowledgements}

This research is supported by an NSERC scholarship and a research grant by Nortel Networks (Wireless Technology Labs), Ottawa.

\section{References}

[1] N.C. Beaulieu and F. Rajwani, "Highly Accurate Simple ClosedForm Approximations to Lognormal Sum Distributions and Densities," IEEE Communications Letters, vol. 8. pp. 709711, December 2004.

[2] J.C.S.S. Filho, P. Cardieri, and M.D. Yacoub, "Simple Accurate Lognormal Approximation to Lognormal Sums," Electronics Letters, vol. 41, pp. 1016-1017, September 2005.

[3] Y.S. Yeh and S.C. Schwartz, "Outage Probability in Mobile Telephony Due to Multiple Log-Normal Interferers," IEEE Transactions on Communications, vol. 32, pp. 380-388, April 1984.

[4] Z. Kostic, I. Maric, and X. Wang, "Fundamentals of Dynamic Frequency Hopping in Cellular Systems," IEEE Journal on Selected Areas in Communications, vol. 19, pp. 2254-2266, November 2001.

[5] O. Mubarek, H. Yanikomeroglu, and S. Periyalwar, "Dynamic Frequency Hopping in Cellular Fixed Relay Networks," Vehicular Technology Conference, Spring 2005, vol. 5, pp. 31123116, May 2005.

[6] M. Chiani, A. Conti, O. Andrisano, "Outage Evaluation for Slow Frequency-Hopping Mobile Radio Systems," IEEE Transactions on Communications, vol. 47, pp. 1865-1874, December 1999. 